\title{
Conservation Importance of Flora in the Kurulu Kele Sanctuary, Sri Lanka
}

\author{
Pemarathne S.K.S. ${ }^{1 *}$ and Gunaratne A.M.T.A. ${ }^{2}$ \\ ${ }^{1}$ Postgraduate institute of Science, University of Peradeniya, Peradeniya, Sri Lanka \\ ${ }^{2}$ Department of Botany, Faculty of Science, University of Peradeniya, Peradeniya, Sri Lanka \\ *kushansp@live.co.uk
}

\begin{abstract}
Of the 3,771 flowering plant species in Sri Lanka 926 are endemic to the country. Nearly $90 \%$ of the endemic flora are concentrated in the wet zone of the island. Kurulu Kele, which is located in the lowland wet zone, was declared as a sanctuary in 1941 due to its high bird and plant diversity Due to its location in the centre of a city, this sanctuary faces lot of human pressures. Two types of habitats identified at the Kurulu Kele Sanctuary (KKS), include the dense forest area and the rocky area. The vegetation sampling was carried out in twelve randomly selected $10 \mathrm{~m} \times 10 \mathrm{~m}$ plots (6 quadrats in each habitat) from December 2011 to June 2012, in order to investigate the floristic composition of the KKS.

A total of 75 species of higher plants belonging to 33 families were recorded. Of the 75 plant species $55 \%$ were trees, $20 \%$ were tree lets and $25 \%$ were shrubs. Twenty five percent of the plant species were endemic, $63 \%$ were native and $12 \%$ were exotic. Four percent were threatened and 9\% were globally threatened. The plant density was 6,867 and 4,617 individuals per ha in the dense forest area and in the rocky area, respectively. The Shannon Wiener Index $(\mathrm{H})$ was higher for the dense forest area (3.50) than the rocky area (3.34).
\end{abstract}

The dominant families in the dense forest area were Anacardiaceae, Euphorbiaceae and Moraceae while in the rocky area the dominant families were Anacardiaceae, Euphorbiaceae, Clusiaceae and Rutaceae. Only two endemic tree species; Strychnos benthamii (Loganiaceae) and Artocarpus nobilis (Moraceae) were recorded in both habitat types.

In the KKS, $65 \%$ of the individuals belonged to $10 \mathrm{~cm}-39 \mathrm{~cm}$ GBH class. Densities of the other GBH classes were very low, which is a characteristic of a disturbed forest. Fourty two percent of the individuals in the dense forest area belonged to $10 \mathrm{~cm}-39 \mathrm{~cm} \mathrm{GBH}$ class, while twenty one percent of the individuals in the rocky area belonged to $10 \mathrm{~cm}-39 \mathrm{~cm} \mathrm{GBH}$ class.

The majority of the remaining forest in the wet zone of Sri Lanka are fragmented and degraded further due to development projects, wood extraction and over exploitation of forest products. Although KKS had an extent of 109 ha in 1941, due to various human impacts KKS is now left with only 11.32 ha $(10.4 \%)$. Therefore, immediate action should be taken to conserve this lowland forest fragment as it provides many ecological and economical services. There is a great potential to develop KKS as an ecotourism destination due to its high biodiversity and the culture of people living in the surrounding area.

Keywords: Plant diversity, Floristic composition, Plant density 